\title{
BIFURCATION OF MINIMAL SURFACES IN RIEMANNIAN MANIFOLDS
}

\author{
JÜRGEN JOST, XIANQING LI-JOST, AND XIAO-WEI PENG
}

\begin{abstract}
We study the bifurcation of closed minimal surfaces in Riemannian manifolds through higher order variations of the area functional and relate it to elementary catastrophes.
\end{abstract}

\section{INTRODUCTION}

In this paper, we study how geodesics and, more generally, minimal submanifolds that are degenerate in the sense that they admit nontrivial Jacobi fields bifurcate if we vary the underlying Riemannian metric. It turns out that if the metric is for example real analytic, the bifurcation behaviour can be described rather explicitly in terms of a polynomial. If suitable conditions are satisfied, one can detect the elementary catastrophes of catastrophe theory (see [BR]).

A bifurcation and catastrophe analysis so far has been carried out only for solutions of Plateau's problem in Euclidean space. Beeson-Tromba [BT] detected the cusp catastrophe in the bifurcation of Enneper's minimal surface under changes of the boundary curve. Büch [BÜ] gave certain conditions on the Weierstra $\beta$ representation of a minimal surface that imply the bifurcation behaviour of catastrophe theory under changes of the boundary. These conditions seem to be hard to verify and not very naturally adapted to the problem. X. LiJost $[\mathrm{LJ}]$ developed a more natural and general approach by studying foliations of Riemannian manifolds through minimal surfaces with varying boundary and using an implicit function theorem argument the basic idea of which goes back to Lichtenstein [LI].

In the present setting, such foliations do not exist any more because we study closed minimal submanifolds and vary the underlying Riemannian metric. Nevertheless, we adapt and generalize the implicit function theorem argument of [LJ].

We hope that the methods of the present paper can be used in the theory of closed geodesics in order to show the existence of infinitely many of them for certain nongeneric metrics by perturbing the metric to a generic one where this is known (see [KL], [HI]).

Received by the editors March 24, 1992.

1991 Mathematics Subject Classification. Primary 53A10, 58E12, 58G25.

Key words and phrases. Minimal submanifolds, bifurcation.

The authors were supported by SFB 237, SFB 256, and Procope.

(c) 1995 American Mathematical Society 
We thank the referee for his careful comments.

\section{NOTATION AND PRELIMINARIES}

$N$ denotes an $n$-dimensional compact oriented manifold with Riemannian metric $g_{0}, \quad M$ an $m$-dimensional compact oriented immersed minimal submanifold (without boundary) of $N . M$ is equipped with the metric induced from $g_{0}$, denoted by $\gamma=i^{*}\left(g_{0}\right)$, where $i: M \rightarrow N$ is the inclusion. $x^{1}, \ldots, x^{m}$ denote local coordinates on $M$. The volume element then is

$$
\operatorname{dvol}(M):=\operatorname{dvol}(M, \gamma)=\sqrt{\operatorname{det}\left(\gamma_{i j}\right)} d x^{1} \wedge \cdots \wedge d x^{m},
$$

and the volume of $(M, g)$ is

$$
\operatorname{Vol}(M):=\operatorname{Vol}(M, \gamma)=\int_{M} \sqrt{\operatorname{det}\left(\gamma_{i j}\right)} d x^{1} \wedge \cdots \wedge d x^{m} .
$$

Let $\Phi_{t}: N \rightarrow N$ be a differentiable family of diffeomorphisms with $\Phi_{0}=\mathrm{id}_{N}$. $X=\left.\frac{d \Phi_{1}}{d t}\right|_{t=0}$ is the variation vector field on $N$. The first variation of $\operatorname{Vol}(M)$ is given by

$$
\left.\frac{d}{d t} \operatorname{Vol}\left(\Phi_{t}(M)\right)\right|_{t=0}=-\int_{M}\langle H, X\rangle \operatorname{dvol}(M),
$$

where $H$ is the mean curvature of $M$ in $N$ defined by

$$
H=\operatorname{tr}_{g} B \text {, }
$$

and $B$ is the second fundamental form of $M . M$ is minimal if and only if $H \equiv 0$.

The second variation of $\operatorname{Vol}(M)$ is given by

$$
\left.\frac{d^{2}}{d t^{2}} \operatorname{Vol}\left(\Phi_{t}(M)\right)\right|_{t=0}=\int_{M}\langle J X, X\rangle \operatorname{dvol}(M),
$$

where $J$ is the Jacobi operator of $M$ defined by

$$
J(X)=-\Delta X+R(X)-B(X) .
$$

In order not to have to consider the trivial case of tangential variations (which do not change the volume of $M$ ), we assume that all variational vector fields $X=\left.\frac{d \Phi_{1}}{d t}\right|_{t=0}$ satisfy in the sequel for all $x \in M$

$$
X(x) \in \nu_{x} M:=\left(T_{x} M\right)^{\perp},
$$

i.e., $X$ is always normal to $M$.

If $\operatorname{codim} M=1$ and $\vec{n}$ is a unit normal vector field of $M$ and $X=\xi \vec{n}$, then the second variation can be written as

$$
\left.\frac{d^{2}}{d t^{2}} \operatorname{Vol}\left(\Phi_{t}(M)\right)\right|_{t=0}=\int_{M}\left(|\nabla \xi|^{2}-\left(\operatorname{Ric}(\vec{n})+|B|^{2}\right) \xi^{2}\right) \operatorname{dvol}(M) .
$$

The preceding formulae are well known. A proof can be found, e.g., in [LJ].

Obviously, $J$ is a second order elliptic operator on the space of sections of the normal bundle of $M$ with respect to the induced connection $\nabla$. In case $\operatorname{codim} M=1, J$ can be viewed as an elliptic operator on the functions of $M$. (see [LA]). 
For technical purposes, we want to identify the normal bundle $\nu M$ of $M$ in $N$ with the normal bundle of the varying submanifold $\Phi_{t} M$ in $N$.

Let exp be the exponential map of $N$ with respect to the Riemannian metric $g_{0}$. exp : $U \rightarrow N$ is a local diffeomorphism, where $U$ is a local neighborhood of the zero section of $\nu M$. Let $\Gamma^{s}(\nu M)$ be the space of all sections of $\nu M$ of Sobolev class $H^{s}$. We choose $s$ so large that all sections appearing in the sequel will be of class $C^{2}$. For $\varphi \in \Gamma^{s}(\nu M)$, we define a submanifold of $N$ by

$$
M_{\varphi}=\left\{\exp _{p} \varphi(p) \mid p \in M\right\} .
$$

For $\varphi$ small enough with respect to the $H^{s}$-norm, $M_{\varphi}$ is diffeomorphic to $M$ via exp.

Now we try to identify $\nu M$ and $\nu M_{\varphi}$. For any $p \in M$ we transfer $\nu_{p} M$ via parallel transport $P$ along the geodesic $\exp _{p} t \varphi(p)$ to $T_{q} N$, where $q=$ $\exp _{p} \varphi(p) \in M_{\varphi} . P\left(\nu_{p} M\right)$ is transversal to the tangent space $T_{q} M_{\varphi}$ of $M_{\varphi}$ at $q$. $\nu_{q} M_{\varphi}$ is always transversal to $T_{q} M_{\varphi}$. For any $\xi \in \nu_{q} M_{\varphi}$, we project $\xi$ to $P\left(\nu_{p} M\right)$ and then parallel transport it back to $\nu_{p} M$. We denote this procedure by

$$
f_{\varphi}: \nu M_{\varphi} \rightarrow \nu M .
$$

$f_{\varphi}$ is an isomorphism. Moreover, $f_{\varphi}$ depends differentiably on $\varphi$.

We denote the mean curvature vector field of $M_{\varphi}$ by $H_{\varphi} \in \Gamma^{s-2}\left(\nu M_{\varphi}\right)$. $f_{\varphi}\left(H_{\varphi}\right)$ then is a normal vector field on $M$. We can thus define the operator

$$
\begin{aligned}
\Lambda_{g_{0}}: \Gamma^{s}(\nu M) & \rightarrow \Gamma^{s-2}(\nu M), \\
\varphi & \mapsto \Lambda_{g_{0}}(\varphi):=f_{\varphi}\left(H_{\varphi}\right) .
\end{aligned}
$$

We now differentiate $\Lambda_{g_{0}}$ at the zero section, using the assumption that $M$ is minimal, i.e., $H \equiv 0$. Let $\psi \in \Gamma^{s}(\nu M)$. Then

$$
d \Lambda_{g_{0}}(\psi)=\left.\frac{d}{d t} f_{t \psi}\left(H_{t \psi}\right)\right|_{t=0}=J(\psi),
$$

since $H_{0} \equiv 0$ (mean curvature of $M$ ) and $f_{0}=$ id.

$J$ is a Fredholm operator from $\Gamma^{s}(\nu M)$ to $\Gamma^{s-2}(\nu M)$. If $\operatorname{Ker} J=\{0\}$, then $J$ is an isomorphism. $J$ depends on the Riemannian metric $g_{0}$, and we therefore also write $J_{g_{0}}$ instead of $J$.

Let $\mathscr{M}^{\ell}$ be the space of all Riemannian metrics of Sobolev class $H^{\ell}$ on $N$. We consider the following differentiable map

$$
\begin{aligned}
\tilde{\Lambda}: \Gamma^{s}(\nu M) \times \mathscr{M}^{s+1} & \rightarrow \Gamma^{s-2}(\nu M), \\
(\varphi, g) & \mapsto \Lambda_{g}(\varphi) .
\end{aligned}
$$

$\tilde{\Lambda}$ satisfies $\tilde{\Lambda}\left(0, g_{0}\right)=0$. If $\operatorname{Ker} J=\{0\}$, then $\frac{\partial \tilde{\Lambda}}{\partial \varphi}\left(0, g_{0}\right)=d \Lambda_{g_{0}}$ is an isomorphism.

The implicit function theorem implies that there exist a neighborhood $U\left(g_{0}\right)$ of $g_{0}$ in $\mathscr{M}^{s+1}$ and a neighborhood $V$ of the zero section in $\Gamma^{s}(\nu M)$, and a differentiable map

$$
\varphi: U\left(g_{0}\right) \rightarrow V \subset \Gamma(\nu M)
$$


such that

$$
\tilde{\Gamma}(\varphi(g), g)=0, \text { for all } g \in U\left(g_{0}\right) .
$$

This means (cf. (2.8))

$$
f_{\varphi(g), g}\left(H_{\varphi(g), g}\right)=0 \text {, }
$$

hence $H_{\varphi(g), g}=0$, since $f_{\varphi(g), g}$ is an isomorphism.

We thus have the following well-known result:

Proposition. If $\operatorname{Ker} J=\{0\}$, then for any Riemannian metric $g$ close enough to $g_{0}$ there exists a minimal submanifold $M_{\varphi}$ with respect to $g$ close to $M$ (in particular diffeomorphic to $M$ ).

Here "close" refers to the above Sobolev space $\mathscr{M}^{s+1}$.

\section{JACOBI FIELDS AND BIFURCATION OF MINIMAL IMMERSIONS}

As before, $i: M \rightarrow N$ is a minimal immersion with Jacobi operator $J$. Since $J$ is elliptic, $K:=\operatorname{Ker} J$ is a finite dimensional vector space consisting of smooth vector fields.

On $\Gamma^{s}(\nu M)$, we have the $L^{2}$-metric induced from the Riemannian metric go. We let

$$
\operatorname{Pr}: \Gamma^{s}(\nu M) \rightarrow K
$$

be the orthogonal projection w.r.t. this $L^{2}$-metric. Define

$$
\begin{aligned}
L_{g_{0}}: \Gamma^{s}(\nu M) & \rightarrow \Gamma^{s-2}(\nu M), \\
\varphi & \mapsto f_{\varphi}\left(H_{\varphi}\right)+\operatorname{Pr}(\varphi) .
\end{aligned}
$$

Lemma 1. $L_{g_{0}}$ is a local diffeomorphism.

Proof. $L_{g_{0}}$ is continuously differentiable. We compute the derivative at the zero section. For $\psi \in \Gamma^{s}(\nu M)$

$$
d L_{g_{0}}(\psi)=\left.\frac{d}{d t} L_{g_{0}}(t \psi)\right|_{t=0}=J_{g_{0}}(\psi)+\operatorname{Pr}(\psi)
$$

Let $J_{g_{0}}^{*}=\Gamma^{s-2}(\nu M) \rightarrow \Gamma^{s}(\nu M)$ be the adjoint operator of $J_{g_{0}}$. Since $J_{g_{0}}$ is formally selfadjoint with respect to the $L^{2}$-metric and since the elements of the kernel of $J_{g_{0}}$ are smooth, we may identify $\operatorname{Ker} J_{g_{0}}$ and $\operatorname{Ker} J_{g_{0}}^{*}$. We then have the orthogonal decomposition

$$
\Gamma^{s-2}(\nu M)=\operatorname{Ker} J_{g_{0}} \oplus \operatorname{Im} J_{g_{0}} .
$$

Thus, if $d L_{g_{0}}(\psi)=0$, then also $J_{g_{0}}(\psi)=0$ and $\operatorname{Pr}(\psi)=0$. Hence $\psi=0$, and consequently $d L_{g_{0}}$ is injective. Since $J_{g_{0}}$ is a Fredholm operator, $d L_{g_{0}}$ is also surjective, and the inverse is also continuous.

It follows from Lemma 1 that there exists a neighborhood $U\left(g_{0}\right)$ of $g_{0}$ in $\mathscr{M}^{s+1}$ with the property that, for any $g \in U\left(g_{0}\right)$, the operator $L_{g}$ is a local diffeomorphism. We consider

$$
\begin{aligned}
L: \Gamma^{s}(\nu M) \times \mathscr{M}^{s+1} & \rightarrow \Gamma^{s-2}(\nu M), \\
(\varphi, g) & \mapsto L_{g}(\varphi)=f_{\varphi, g}\left(H_{\varphi, g}\right)+\operatorname{Pr}_{g_{0}}(\varphi) .
\end{aligned}
$$


Since $L\left(0, g_{0}\right)=0$, it follows that there exist neighborhoods $U_{1}$ and $U_{2}$ of 0 in $\Gamma^{s}(\nu M)$ and $\Gamma^{s-2}(\nu M)$, resp., with the property that for any $g \in U\left(g_{0}\right)$ and any $\xi \in U_{2}$, there exists $u \in U_{1}$ with $L(u, g)=\xi$, i.e.,

$$
f_{u, g}\left(H_{u, g}\right)+\operatorname{Pr}_{g_{0}}(u)=\xi .
$$

$u$ depends differentiably on $g$ and $\xi$; we write $u=u(\xi, g)$. Thus, our equation becomes

$$
L(u(\xi, g), g)=\xi .
$$

Our aim now is to find $\xi$ with

$$
\operatorname{Pr}_{g_{0}}(u(\xi, g))=\xi,
$$

because by (3.2) this is equivalent to

$$
f_{u, g}\left(H_{u, g}\right)=0 \Longleftrightarrow H_{u, g}=0,
$$

meaning that $u$ is a minimal submanifold with respect to the metric $g$ on $N$. It will turn out that this is possible for suitable metrics $g$.

Some notation: $k:=\operatorname{dim} K, \xi_{1}, \ldots, \xi_{k}$ is an orthonormal basis of $K$ with respect to the $L^{2}$-metric. For $\xi \in K$, we write

$$
\xi=\sum_{i=1}^{k} \nu_{i} \xi_{i} \quad\left(\nu_{i} \in \mathbb{R}\right) .
$$

(3.4) is equivalent to

$$
\sum_{i=1}^{k}\left(u, \xi_{i}\right) \xi_{i}=\sum_{i=1}^{k} \nu_{i} \xi_{i}
$$

or

$$
\left(u, \xi_{i}\right)=\nu_{i} \quad \text { for } i=1, \ldots, k
$$

We write

$$
u(\xi, g)=u\left(\nu_{1}, \ldots, \nu_{k} ; g\right)
$$

and put

$$
u_{i}:=\frac{\partial u}{\partial \nu_{i}}\left(0, \ldots, 0 ; g_{0}\right), \quad u_{g}:=\frac{\partial u}{\partial g}\left(0, \ldots, 0 ; g_{0}\right) .
$$

Differentiating (3.5) with respect to $\nu_{j}$ yields

(3.3) is written as

$$
\left(u_{j}, \xi_{i}\right)=\delta_{i j} \quad \text { for } i, j=1, \ldots, k .
$$

$$
L\left(u\left(\nu_{1}, \ldots, \nu_{k} ; g\right), g\right)=\sum_{i=1}^{k} \nu_{i} \xi_{i} .
$$

Differentiating (3.6) with respect to $\nu_{j}$ yields

$$
J_{g_{0}}\left(u_{j}\right)+\operatorname{Pr}_{g_{0}}\left(u_{j}\right)=\xi_{j} \quad \text { for } j=1, \ldots, k .
$$

Since $\xi_{j} \in K=\operatorname{ker} J_{g_{0}}$, we also have

$$
J_{g_{0}}\left(\xi_{j}\right)+\operatorname{Pr}_{g_{0}}\left(\xi_{j}\right)=\xi_{j} .
$$


By Lemma $1 d L_{g_{0}}=J_{g_{0}}+\operatorname{Pr}_{g_{0}}$ is injective, and comparing (3.7) and (3.8) thus implies

$$
\xi_{j}=u_{j} \quad \text { for } j=1, \ldots, k .
$$

Lemma 2. For every $\lambda=\left(\lambda_{1}, \ldots, \lambda_{k}\right) \in \mathbb{R}^{k}$, there exists $h \in \Gamma^{s+1}\left(T^{*} N \otimes T^{*} N\right)$ with

$$
\left(u_{g} h, \xi_{i}\right)=\lambda_{i} \quad \text { for } i=1, \ldots, k .
$$

Proof. We let $g_{t}=g_{0}+t h$ be the variation of $g_{0}$ induced by $h$. For simplicity of notation, we shall write $g$ instead of $g_{t}$. From (3.2)

$$
\left(f_{u, g}\left(H_{u, g}\right), \xi_{i}\right)+\left(\operatorname{Pr}_{g_{0}}(u), \xi_{i}\right)=\left(\xi, \xi_{i}\right) .
$$

Differentiating (3.11) with respect to $t$ at $t=0, \nu=0$ gives

$$
\frac{d}{d t}\left(f_{u, g}\left(H_{u, g}\right), \xi_{i}\right)+\frac{d}{d t}\left(\operatorname{Pr}_{g_{0}}(u), \xi_{i}\right)=0 .
$$

Hence

$$
\left(u_{g} h, \xi_{i}\right)=-\left(\frac{d}{d t} H_{u, g}, \xi_{i}\right) .
$$

We let $\left(x^{1}, \ldots, x^{n}\right)$ be local coordinates on $N$ such that $\left(x^{1}, \ldots, x^{m}, 0, \ldots\right.$, $0)$ yield local coordinates of $M$.

We let $e_{1}, \ldots, e_{m}$ be an orthonormal basis of $T M$ with respect to $g_{0}$, and write

$$
e_{j}=a_{j}^{\alpha} \frac{\partial}{\partial x^{\alpha}} \quad(j=1, \ldots, m)
$$

Thus

$$
\delta_{i j}=a_{i}^{\alpha} a_{j}^{\beta} g_{\alpha \beta}
$$

and

$$
g^{\alpha \beta}=a_{j}^{\alpha} a_{j}^{\beta}
$$

Then

$$
\left(H_{u, g_{0}}, \xi_{i}\right)=\left(\nabla_{e_{j}} e_{j}, \xi_{i}\right)
$$

since $\xi_{i}$ is normal to $M$.

We now let all these constructions depend on $t$, i.e., we compute the mean curvature vector of the submanifold defined by $u\left(0, g_{t}\right)$. We thus have to compute

$$
\frac{d}{d t}\left(\nabla_{e_{j}^{t}}^{t} t_{j}^{t}, \xi_{i}\right)_{\mid t=0}
$$

Here

$$
\epsilon_{j}^{t}=a_{j}^{\alpha}\left(u\left(0, g_{t}\right), g_{t}\right) \frac{\partial}{\partial x^{\alpha}} .
$$

$e_{j}^{t}$ varies in $t$ both because the immersed submanifold $u$ varies and the orthonormalization process depends on the metric $g_{t}$. We shall choose $h$ in such a way, however, that along $M$, defined by $u\left(0, g_{0}\right), g_{t}$ will coincide with $g_{0}$ so that the second dependence on $t$ will play no role. 
Therefore, for such variations $g_{t}$,

$$
\begin{aligned}
\left.\frac{d}{d t}\left(\nabla_{e_{j}^{t}}^{t} e_{j}^{t}, \xi_{i}\right)\right|_{t=0} & =\left.\frac{d}{d t}\left(a_{j}^{\alpha} \frac{\partial \alpha_{j}^{\beta}}{\partial x^{\alpha}} \frac{\partial}{\partial x^{\beta}}+a_{j}^{\alpha} a_{j}^{\beta} \nabla_{\frac{\partial}{\partial x^{\alpha}}}^{t} \frac{\partial}{\partial x^{\beta}}, \xi_{i}\right)\right|_{t=0} \\
& =\left(J_{g_{0}}\left(u_{g} h\right), \xi_{i}\right)+\left(g^{\alpha \beta} \frac{d}{d t} \Gamma_{\alpha \beta_{l t=0}^{\gamma}}^{\gamma} \frac{\partial}{\partial x^{\gamma}}, \xi_{i}\right)
\end{aligned}
$$

where the first term represents the variation of the submanifold and the second one that of the connection.

Since $\xi_{i} \in \operatorname{ker} J_{g_{0}}$ and $J_{g_{0}}$ is selfadjoint, the first term on the right-hand side of (3.14) vanishes. The second is

$$
\int_{M} \frac{1}{2} g^{\alpha \beta} g^{\gamma \delta}\left(h_{\alpha \delta, \beta}+h_{\beta \delta, \alpha}+h_{\alpha \beta, \delta}\right)\left\langle\frac{\partial}{\partial x^{\gamma}}, \xi_{i}\right\rangle \operatorname{dvol}(M) .
$$

By taking the exponential map of the normal bundle $\nu M$, we may choose our coordinates $\left(x^{1}, \ldots, x^{n}\right)$ in such a way that

$$
\begin{array}{ll}
g_{r s}\left(x^{1}, \ldots, x^{m}, 0, \ldots, 0\right)=\delta_{r s} & \text { for } r, s=m+1, \ldots, n, \\
g_{a s}\left(x^{1}, \ldots, x^{m}, 0, \ldots, 0\right)=0 & \text { for } a=1, \ldots, m, s=m+1, \ldots, n .
\end{array}
$$

Of course, the dummy index $s$ used here has nothing to do with the Sobolev space index $s$ used throughout the paper.

The expression in (3.15) then reduces to

$$
\int_{M} \frac{1}{2} g^{\alpha \beta}\left(h_{\alpha s, \beta}+h_{\beta s, \alpha}-h_{\alpha \beta, s}\right)\left\langle\frac{\partial}{\partial x^{s}}, \xi_{i}\right\rangle \operatorname{dvol}(M),
$$

since $\xi_{i}$ is normal to $M$. The summation with respect to $s$ is only from $m+$ $1, \ldots, n$, again since $\xi_{i}$ is normal to $M$.

We now choose

$$
h_{\alpha \beta}= \begin{cases}\frac{2}{m} x^{s} \lambda_{i} g_{\alpha \beta} \frac{1}{\int_{M}\left|\xi_{i}\right|} & \text { for } \alpha, \beta=1, \ldots, m, \\ 0 & \text { otherwise. }\end{cases}
$$

Expression (3.16) then becomes

$$
-\frac{\lambda_{i}}{\int_{M}\left|\xi_{i}\right|} \int_{M} \sum_{s=m+1}^{n}\left\langle\frac{\partial}{\partial x^{s}}, \xi_{i}\right\rangle \operatorname{dvol}(M)=-\lambda_{i},
$$

since $\frac{\partial}{\partial x^{s}}, s=m+1, \ldots, n$, is an orthonormal basis of $\nu M$ along $M$.

Putting everything together, we obtain $\left(u_{g} h, \xi_{i}\right)=\lambda_{i}$.

We consider the Taylor development of $\left(u, \xi_{i}\right)$. From (3.5)

$$
\begin{aligned}
\nu_{i}= & \left(u, \xi_{i}\right)=\left(u\left(0, g_{0}\right), \xi_{i}\right)+\sum_{j}\left(u_{j}, \xi_{i}\right) \nu_{j} \\
& +\frac{1}{2} \sum_{l, m}\left(u_{l m}, \xi_{i}\right) \nu_{l} \nu_{m}+\text { higher order terms in } \nu \text { only } \\
& +\left(u_{g} h, \xi_{i}\right)+\frac{1}{2} \sum_{j}\left(u_{j g} h, \xi_{i}\right) \nu_{j}+\frac{1}{2}\left(u_{g g} h h, \xi_{i}\right) \\
& + \text { terms of higher order in both } \nu \text { and } h .
\end{aligned}
$$


Because $u\left(0, g_{0}\right)=0$ and $u_{j}=\xi_{j}(3.18)$ becomes

$$
\begin{aligned}
0= & \left(u_{g} h, \xi_{i}\right)+\frac{1}{2} \sum_{l, m}\left(u_{\ell m}, \xi_{i}\right) \nu_{\ell} \nu_{m}+\text { higher order terms in } \nu \text { only } \\
& +\frac{1}{2} \sum_{j}\left(u_{j g} h, \xi_{i}\right) \nu_{j}+\text { terms of higher order in both } \nu \text { and } h .
\end{aligned}
$$

We write this also as

$$
0=\left(u_{g} h, \xi_{i}\right)+Q^{i}(\nu)+R^{i}(\nu, h)
$$

where

$$
\begin{aligned}
Q^{i}(\nu)= & \frac{1}{2} \sum_{\ell, m}\left(U_{\ell m}, \xi_{i}\right) \nu_{\ell} \nu_{m} \\
& + \text { higher order terms in } \nu \text { only }, \\
R^{i}(\nu, h)= & \frac{1}{2} \sum_{j}\left(U_{j g} h, \xi_{i}\right) \nu_{j}
\end{aligned}
$$

+ higher order terms in both $\nu$ and $h$.

In order to interpret the terms in (3.19), we differentiate (3.6) twice with respect to $\nu$ at $\nu=0$, obtaining with the help of (3.9)

$$
d J_{g_{0}}\left(\xi_{\ell}, \xi_{m}\right)+J_{g_{0}} u_{\ell m}+\sum_{j}\left(u_{\ell m}, \xi_{j}\right) \xi_{j}=0 .
$$

Multiplying with $\xi_{i}$ yields, since $J_{g_{0}} \xi_{i}=0$,

$$
\left(u_{\ell m}, \xi_{j}\right)=-\left(d J_{g_{0}}\left(\xi_{\ell}, \xi_{m}\right), \xi_{j}\right) \text {. }
$$

Thus $\left(u_{\ell m}, \xi_{j}\right)$ can be expressed by the third variation of volume of our submanifold. Similarly, if all $u_{\ell m}$ vanish, i.e., if the third variation vanishes on all Jacobi fields, then the $\left(u_{\ell m n}, \xi_{j}\right)$ can be expressed by the fourth variation, and so on.

We now determine the leading part of $Q^{i}(\nu)$ as follows:

Let $n_{i}$ be the smallest positive integer with the property that there exist positive integers $\mu_{i 1}, \ldots, \mu_{i k}$ satsifying the following:

If we put

$$
\nu_{j}=t_{i}^{\mu_{i j}} \tau_{j}
$$

then we have with $\tau=\left(\tau_{1}, \ldots, \tau_{k}\right)$

$$
Q^{i}(\nu)=t_{i}^{n_{i}} P^{i}(\tau)+o\left(t_{i}^{n_{i}}\right)
$$

with a polynomial $P^{i}(\tau)$ which is nondegenerate in the sense that

$$
\frac{\partial}{\partial \tau_{j}} P^{i}(\tau) \not \equiv 0 \text { for all } j=1, \ldots, k \text {. }
$$

If such $n_{i}$ exists, we say that $Q^{i}(\nu)$ is nondegenerate.

We call $\lambda=\left(\lambda_{1}, \ldots, \lambda_{k}\right) \in \mathbb{R}^{k}$ critical if there exists $\tau^{0}=\left(\tau_{1}^{0}, \ldots, \tau_{k}^{0}\right)$ with

$$
\lambda^{i}+P^{i}\left(\tau_{1}^{0}, \ldots, \tau_{k}^{0}\right)=0
$$


and

$$
\frac{\partial\left(P^{1}, \ldots, P^{k}\right)}{\partial\left(\tau_{1}, \ldots \tau_{k}\right)}\left(\tau^{0}\right)=0
$$

If $\lambda$ is not critical, it is called regular. The set of critical $\lambda$ has measure 0 , for example Sard's theorem.

Theorem 1. Suppose that in the Taylor expansion (3.20) all $Q^{i}(\nu), i=1, \ldots$, $k$, are nondegenerate (as defined after (3.25)). Then for each regular $\lambda=$ $\left(\lambda_{1}, \ldots, \lambda_{k}\right) \in \mathbb{R}^{k}$ there exist $\varepsilon>0$ and a variation $g_{\rho}=g_{0}+\rho \bar{h}$ of the metric $g_{0}$ and for $0<\rho<\varepsilon$, there exists a neighborhood $U_{\rho}$ of 0 in $\Gamma^{s}(\nu M)$ with the property that the number of minimal submanifolds of $N$ of the form $\exp \varphi$ for $\varphi \in U_{\rho}$ equals the number of solutions of

$$
\lambda+P(\tau)=0 \quad\left(P=\left(P^{1}, \ldots, P^{k}\right)\right) .
$$

Theorem 1 says that, generically, the bifurcation behaviour of the minimal submanifold $M$ is governed by the solutions of a polynomial equation. As $\rho \rightarrow 0, \quad U_{\rho}$ will shrink to 0 .

The assumption of Theorem 1 is satisfied if $g_{0}$ is real analytic (which implies that $M$ is a real analytic submanifold of $N)$ and $M$ is not contained in a oneparameter family of minimal submanifolds. Namely, in this case the $Q^{i}(\nu)$ are real analytic and therefore have Taylor expansions in the variables $\nu_{1}, \ldots, \nu_{k}$. Excluding that $M$ is contained in a family of minimal submanifolds guarantees that this expansion is nondegenerate as defined in (3.25).

Proof of Theorem 1. We have to determine the number of solutions of (3.20). The strategy is to replace $Q^{i}$ by $P^{i}$, suitably scaled, and disregard $R^{i}$ in the first step, where $L$ is chosen in such a way that, up to a scaling factor,

$$
\left(u_{g} h, \xi_{i}\right)=\lambda_{i} \quad(i=1, \ldots, k) \text {. }
$$

An application of the implicit function theorem will show that the number of solutions is not affected by this simplification of the equation.

As in (3.23) we put

$$
\nu_{j}=t_{i}^{\mu_{i j}} \tau_{i j} \quad \text { with } \tau_{i}=\left(\tau_{i 1}, \ldots, \tau_{i k}\right) \in \mathbb{R}^{k}, t=\left(t_{1}, \ldots, t_{k}\right) \in \mathbb{R}^{k},
$$

and

$$
h=t_{t}^{n_{i}} \bar{h}
$$

We now let

$$
m_{i}:=\frac{1}{n_{i}} \prod_{j=1}^{k} n_{j}, \quad \bar{m}:=\prod_{j=1}^{k} n_{j}
$$

and

$$
t_{i}=t^{m_{i}}
$$

Equation (3.28) becomes

$$
h=t^{\bar{m}} \bar{h},
$$

and (3.27) is

$$
\nu_{j}=t^{m_{i} \mu_{i j}} \tau_{i j}=: t^{\bar{\mu}_{i j}} \tau_{i j}
$$


According to Lemma 2 , there exists $\bar{h}$ with

$$
\left(u_{g} \bar{h}, \xi_{i}\right)=\lambda_{i}, \quad i=1, \ldots, k .
$$

With these conventions, (3.20) becomes

$$
0=t^{\bar{m}} \lambda_{i}+t^{\bar{m}} P^{i}(\tau)+O\left(t^{\bar{m}+1}\right) \quad(i=1, \ldots, k)
$$

which is equivalent to

$$
0=\lambda_{i}+P^{i}(\tau)+O(t) \quad(i=1, \ldots, k) .
$$

We consider (3.26)

$$
0=\lambda+P(\tau)
$$

Since $\lambda$ is regular, (3.34) has at most finitely many solutions $\tau^{(1)}, \ldots, \tau^{(s)}$, for $r=1, \ldots, s$. The Jacobian satisfies

$$
\frac{\partial P}{\partial \tau}\left(\tau^{(r)}\right) \neq 0
$$

We now write (3.33) as

$$
0=\lambda+P(\tau)+S(t, \tau, \bar{h})=: F(t, \tau, \bar{h})
$$

with $S(t, \tau, \bar{h})=O(t)$ for every fixed $(\tau, \bar{h})$, in particular $S(0, \tau, \bar{h})=0$.

$F$ satisfies

$$
F\left(0, \tau^{(r)}, \bar{h}\right)=0 \text { for } r=1, \ldots, s
$$

and by (3.35) the Jacobian satisfies

$$
\frac{\partial F}{\partial \tau}\left(0, \tau^{(r)}, \bar{h}\right) \neq 0 \quad \text { for } r=1, \ldots, s .
$$

The implicit function theorem then implies that there exist functions $\tau^{(r)}(t)$ : $\left(-\varepsilon^{\prime}, \varepsilon^{\prime}\right) \rightarrow \mathbb{R}, r=1, \ldots, s,|t|<\varepsilon^{\prime}$ for some $\varepsilon^{\prime}>0$, with

$$
F\left(t, \tau^{(r)}(t), \bar{h}\right)=0,
$$

which is equivalent to (3.4) (for $t \neq 0$ ), which we wanted to solve, for the variation of metrics

$$
g_{t}=g_{0}+t^{\bar{m}} \bar{h}
$$

(see (3.5), (3.18), (3.20), (3.33)).

We put $\rho:=t^{\bar{m}}$ and prove the theorem.

\section{Special cases and catastrophes}

It is instructive to consider some special cases of Theorem 1. In this section, we assume that the space of Jacobi fields of $M$ is one-dimensional, i.e.,

$$
k=\operatorname{dim} \operatorname{ker} J=1 .
$$

This means that we can drop indices (see, e.g., (3.18)) and that equations like (3.26) became scalar instead of vectorial.

Equation (3.19) becomes

$$
0=\left(u_{g} h, \xi\right)+\frac{1}{2}\left(u_{\nu \nu}, \xi\right) \nu^{2}+\text { higher order terms, }
$$


where $u_{\nu \nu}$ denotes the second derivative of $u$ with respect to $\nu$. The first case we want to discuss is

$$
\left(u_{\nu \nu}, \xi\right) \neq 0
$$

i.e., the third variation of colume of $M$ in the direction $\xi$ does not vanish (see (3.22)).

With $p:=\frac{1}{2}\left(u_{\nu \nu}, \xi\right), h=t^{2} \bar{h}, \nu=t \tau, \lambda=\left(u_{g} \bar{h}, \xi\right)$, (4.2) becomes

$$
0=\lambda t^{2}+p t^{2} \nu^{2}+t^{2} S(t, \nu, \bar{h})
$$

with

$$
S(t, \nu, \bar{h})=O(t) \text { for fixed } \nu, \bar{h}
$$

as before.

For $t \neq 0$, this again is equivalent to

$$
0=\lambda+p \nu^{2}+S(t, \nu, \bar{h}) \text {. }
$$

Thus for $\lambda \neq 0$, depending on the sign of $\frac{\lambda}{p}$, there either exist two solutions of (4.5) or none for sufficiently small $|t|$. This implies for the variation of metrics $g_{\rho}=g_{0}+\rho \bar{h}$ that either for $\rho>0$ there exist two minimal submanifolds, with respect to the metric $g_{\rho}$ near $M$, and none for $\rho<0$, or vice versa, i.e., two solutions for $\rho<0$ and none for $\rho>0$.

This is the simplest possible nontrivial bifurcation process, namely the one for the roots of a cubic polynomial depending on a parameter.

We now discuss the case, again under assumption (4.1), where the third variation vanishes, but the fourth one does not.

$$
\begin{aligned}
\left(u_{\nu \nu}, \xi\right) & =0, \\
\left(u_{\nu \nu \nu}, \xi\right) & \neq 0 .
\end{aligned}
$$

Recalling again $u\left(0, g_{0}\right)=0, u_{\nu}=\xi,(3.19)$ becomes

$$
0=\left(u_{g} h, \xi\right)+\frac{1}{6}\left(u_{\nu \nu \nu}, \xi\right) \nu^{3}+\left(u_{\nu g} h, \xi\right) \nu+\text { higher order terms. }
$$

We now choose a two parameter variation of $g_{0}$ in the directions $h_{1}$ and $h_{2}$. We assume that $h_{1}$ and $h_{2}$ satisfy

(i) $\left(u_{g} h_{1}, \xi\right) \neq 0$.

(ii) $\left(u_{\nu g} h_{2}, \xi\right) \neq 0$, but $\left(u_{g} h_{2}, \xi\right)=0$.

(i) can be achieved by Lemma 2 .

We now put $\nu=t \tau, h=t^{3} \alpha h_{1}+t^{2} \beta h_{2}$ and rewrite (4.8) as

$$
0=t^{3}\left(\left(u_{g} h_{1}, \xi\right) \alpha+\left(u_{\nu g} h_{2}, \xi\right) \beta \tau+\frac{1}{6}\left(u_{\nu \nu \nu}, \xi\right) \tau^{3}+S\left(t, \tau, h_{1}, h_{2}\right)\right)
$$

where again $S\left(t, \tau, h_{1}, h_{2}\right)=O(t)$ for any fixed $\tau, h_{1}, h_{2}$. Theorem 1 implies that, for $t \neq 0$, the solution behavior is qualitatively described by the roots of

$$
0=\left(u_{g} h_{1}, \xi\right) \alpha+\left(u_{\nu g} h_{2}, \xi\right) \beta \tau+\frac{1}{6}\left(u_{\nu \nu \nu}, \xi\right) \tau^{3} .
$$

This is precisely the so-called cusp catastrophe (with $\alpha, \beta$ as parameters). In particular, locally there exist at most three minimal submanifolds in a neighborhood of $M$ with respect to the metric

$$
g_{0}+t^{3} \alpha h_{1}+t^{2} \beta h_{2}
$$


This follows with the reasoning of the proof of Theorem 1. It should now be clear how to detect the other elementary catastrophes (see [BR]) in the present setting.

We remark that an explicit example for the occurrence of a cusp catastrophe in minimal surface theory is Plateau's problem for Enneper's surface. This was described in [BT]. Of course, the setting of Plateau's problem is different from ours. We present here a systematic development so that in specific examples one only has to compute the polynomial $P(t)$ in (3.26) in order to be able to describe the bifurcation behavior.

\section{REFERENCES}

[BR] Th. Bröcker, Differentiable germs and catastrophes, London Math. Soc. Lecture Note Ser. no. 17, Cambridge University Press, London and New York, 1975.

[BT] M.J. Beeson and A.T. Tromba, The cusp catastrophe of Thom in the bifurcation of minimal surfaces, Manuscripta Math. 46 (1984), 273-308.

[BÜ] J. Büch, Bifurkation von Minimalflächen und Elementare Katastrophen, Manuscripta Math. 55 (1986), 269-306.

[HI] N. Hingston, Equivariant Morse theory and closed geodesics, J. Differential Geom. 19 (1984), 85-116.

[KL] W. Klingenberg, Lectures on closed geodesics, Springer-Verlag, Berlin, 1978.

[LA] H.B. Lawson, Lectures on minimal submanifolds, Vol. 1, Publish or Perish, Berkeley, CA, 1980.

[LI] L. Lichtenstein, Untersuchung über zweidimensionale reguläre Variationsprobleme, Monatsh. Math. Phys. 28 (1917).

[LJ] X. Li-Jost, Eindeutigkeit und Verzweigung von Minimalflächen, Dissertation, Univ. Bonn.

RUhr-Universität Bochum, Mathematisches Institut, 44780 Bochum, Germany 